\title{
Boundedness and Lagrange stability of fractional order perturbed system related to unperturbed systems with initial time difference in Caputo's sense
}

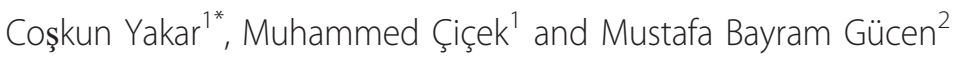

\footnotetext{
* Correspondence: cyakar@gyte. edu.tr

${ }^{1}$ Department of Mathematics, Gebze Institute of Technology, Gebze-Kocaeli 141-41400, Turkey Full list of author information is available at the end of the article
}

\begin{abstract}
In this paper, we have investigated that initial time difference boundedness criteria and Lagrange stability for fractional order differential equation in Caputo's sense are unified with Lyapunov-like functions to establish comparison result. The qualitative behavior of a perturbed fractional order differential equation with Caputo's derivative that differs in initial position and initial time with respect to the unperturbed fractional order differential equation with Caputo's derivative has been investigated. We present a comparison result that again gives the null solution a central role in the comparison fractional order differential equation when establishing initial time difference boundedness criteria and Lagrange stability of the perturbed fractional order differential equation with respect to the unperturbed fractional order differential equation in Caputo's sense.

AMS(MOS) Subject Classification: 34C11; 34D10; 34D99.

Keywords: initial time difference (ITD), boundedness and Lagrange stability, fractional order differential equation, perturbed fractional order differential systems, comparison results
\end{abstract}

\section{Introduction}

The concept of noninteger-order derivative, popularly known as fractional derivative, goes back to the 17 th century [1,2]. It is only a few decades ago, it was realized that the derivatives of arbitrary order provide an excellent framework for modeling the real-world problems in a variety of disciplines from physics, chemistry, biology and engineering such as viscoelasticity and damping, diffusion and wave propagation, electromagnetism, chaos and fractals, heat transfer, electronics, signal processing, robotics, system identification, traffic systems, genetic algorithms, percolation, modeling and identification, telecommunications, irreversibility, control systems as well as economy, and finance $[1,3-5]$.

There has been a surge in the study of the theory of fractional order differential systems, but it is still in the initial stages. We have investigated the boundedness and Lagrange stability of perturbed solution with respect to unperturbed solution with ITD of the nonlinear differential equations of fractional order. The differential operators are

(c) 2011 Yakar et al; licensee Springer. This is an Open Access article distributed under the terms of the Creative Commons Attribution License (http://creativecommons.org/licenses/by/2.0), which permits unrestricted use, distribution, and reproduction in any medium, provided the original work is properly cited. 
taken in the Caputo's sense, we have the relations among the Caputo, RiemannLiouville and Grünwald-Letnikov fractional derivatives, and the initial conditions are specified according to Caputo's suggestion [6], thus allowing for interpretation in a physically meaningful way $[4,5,7]$.

The concept of a Lyapunov function has been employed with great success in a wide variety of investigations to understand qualitative and quantitative properties of dynamic systems for many years. Lyapunov's direct method is a standard technique used in the study of the qualitative behavior of differential systems along with a comparison result $[4,8-11]$ that allows the prediction of behavior of a differential system when the behavior of the null solution of a comparison system is known. The application of Lyapunov's direct method in boundedness theory $[4,9,10,12,13]$ has the advantage of not requiring knowledge of solutions. However, there has been difficulty with this approach when trying to apply it to unperturbed fractional differential systems $[14,15]$ and associated perturbed fractional differential systems with an ITD. The difficulty arises because there is a significant difference between ITD boundedness and Lagrange stability [2,12-20] and the classical notion of boundedness and Lagrange stability for fractional order differential systems $[4,7]$. The classical notions of boundedness and Lagrange stability [5,7-10,21] are with respect to the null solution, but ITD boundedness and Lagrange stability $[2,12-20]$ are with respect to the unperturbed fractional order differential system where the perturbed fractional order differential system and the unperturbed fractional order differential system differ both in initial position and in initial time [2,12-20].

In this work, we have dissipated this complexity and have a new comparison result that again gives the null solution a central role in the comparison fractional order differential system. This result creates many paths for continuing research by direct application and generalization $[15,19,20,22]$.

In Section 2, we present basic definitions, fundamental lemmas and necessary rudimentary material. In Section 3, we have a comparison result in which the stability properties of the null solution of the comparison system imply the corresponding (ITD) boundedness and Lagrange stability properties of the perturbed fractional order differential system with respect to the unperturbed fractional order differential system. In Section 4, we have an example as an application how to apply the main results of main theorems, and in Section 5, we give a conclusion.

\section{Preliminaries}

In this section, we give relation among the fractional order derivatives, Caputo, Reimann-Liouville and Grünwald-Letnikov fractional order derivatives, necessary definition of initial value problems of fractional order differential equations with these sense and a comparison result for Lyapunov-like functions.

\subsection{Fractional order derivatives: Caputo, Reimann-Liouville and Grünwald-Letnikov}

Caputo's and Reimann-Liouville's definition of fractional derivatives, namely,

$$
{ }^{c} D^{q} x=\frac{1}{\Gamma(1-q)} \int_{\tau_{0}}^{t}(t-s)^{-q} x^{\prime}(s) d s, \quad \tau_{0} \leq t \leq T
$$




$$
D^{q} x=\frac{1}{\Gamma(p)}\left(\frac{d}{d t} \int_{\tau_{0}}^{t}(t-s)^{p-1} x(s) d s\right), \tau_{0} \leq t \leq T
$$

respectively order of $0<q<1$, and $p+q=1$ where $\Gamma$ denotes the Gamma function. Fractional derivatives and integrals play an important role in the development of theory of fractional dynamic systems $[4,7,11]$. Using of half-order derivatives and integrals leads to a formulation of certain real-world problems in different areas [1,6]. Fractional derivatives and integrals are also useful in pure mathematics and in applications outside mathematics include such otherwise unrelated topics as: transmission line theory, chemical analysis of aqueous solutions, design of heat-flux meters, rheology of soils, growth of intergranular grooves at metal surfaces, quantum mechanical calculations and dissemination of atmospheric pollutants.

The main advantage of Caputo's approach is that the initial conditions for fractional order differential equations with Caputo derivative take on the same form as that of ordinary differential equations with integer derivatives another difference is that the Caputo derivative for a constant $C$ is zero, while the Riemann-Liouville fractional derivative for a constant $C$ is not zero but equals to $D^{q} C=\frac{C\left(t-\tau_{0}\right)^{-q}}{\Gamma(1-q)}$. By using (2.1.1) and therefore,

$$
{ }^{c} D^{q} x(t)=D^{q}\left[x(t)-x\left(\tau_{0}\right)\right]
$$

and

$$
{ }^{c} D^{q} x(t)=D^{q} x(t)-\frac{x\left(\tau_{0}\right)}{\Gamma(1-q)}\left(t-\tau_{0}\right)^{-q} .
$$

In particular, if $x\left(\tau_{0}\right)=0$, then we obtain

$$
{ }^{c} D^{q} x(t)=D^{q} x(t) .
$$

Hence, we can see that Caputo's derivative is defined for functions for which Riemann-Liouville fractional order derivative exists.

Let us write that Grünwald-Letnikov's notion of fractional order derivative in a convenient form

$$
D_{0}^{q} x(t)=\lim _{\substack{h \rightarrow 0 \\ n h=-t-\tau_{0}}} \frac{1}{h^{q}}[x(t)-S(x, h, r, q)]
$$

where $S(x, h, r, q)=\sum_{r=1}^{n}(-1)^{r+1}\left(\begin{array}{l}q \\ r\end{array}\right) x(t-r h)$ If we know that $x(t)$ is continuous and $\frac{d x(t)}{d t}$ exist and integrable, then Riemann-Liouville and Grünwald-Letnikov fractional order derivatives are connected by the relation

$$
D_{0}^{q} x(t)=D^{q} x(t)=\frac{x\left(\tau_{0}\right)\left(t-\tau_{0}\right)^{-q}}{\Gamma(1-q)}+\int_{\tau_{0}}^{t} \frac{(t-s)^{-q}}{\Gamma(1-q)} \frac{d}{d s} x(s) d s .
$$

By using (2.1.3) implies that we have the relations among the Caputo, Riemann-Liouville and Grünwald-Letnikov fractional derivatives 


$$
{ }^{c} D^{q} x(t)=D^{q}\left[x(t)-x\left(\tau_{0}\right)\right]=D_{0}^{q}\left[x(t)-x\left(\tau_{0}\right)\right]=\frac{1}{\Gamma(1-q)} \int_{\tau_{0}}^{t}(t-s)^{-q} \frac{d x(s)}{d s} d s .
$$

The foregoing equivalent expressions are very useful in the study of qualitative properties of solutions of fractional order differential equations.

\subsection{Fractional order differential equations with Caputo's derivative}

The main advantage of Caputo's approach to fractional derivative is that the initial values and the notion of solution parallel the IVP of differential equations, where the derivative is of order one, that is the usual derivative. Since no known physical interpretation of initial conditions in Riemann-Liouville' s sense $[4,7,11]$ was available, it was felt the solutions obtained are practically useless. Under natural conditions on $x(t)$ as $q \rightarrow n$, the Caputo's derivative becomes the conventional $n$th derivative of $x(t)$ for $n-1<q<n$.

Consider the initial value problems of the fractional order differential equations with Caputo derivative

$$
\begin{aligned}
& { }^{c} D^{q} x=f(t, x), x\left(t_{0}\right)=x_{0} \text { for } t \geq t_{0}, t_{0} \in \mathbb{R}_{+} \\
& { }^{c} D^{q} x=f(t, x), x\left(\tau_{0}\right)=y_{0} \text { for } t \geq \tau_{0}, \tau_{0} \in \mathbb{R}_{+}
\end{aligned}
$$

and the perturbed system of initial value problem of the fractional order differential equation with Caputo's derivative of (2.2.2)

$$
\begin{aligned}
& { }^{c} D^{q} y=F(t, y), y\left(\tau_{0}\right)=y_{0} \text { for } t \geq \tau_{0} \\
& { }^{c} D^{q} w=H(t, w), w\left(\tau_{0}\right)=y_{0}-x_{0} \text { for } t \geq \tau_{0}
\end{aligned}
$$

where $f, F, H \in C\left[\left[t_{0}, \tau_{0}+T\right] \times \mathbb{R}^{n}, \mathbb{R}^{n}\right]$; satisfy a local Lipschitz condition on the set $\mathbb{R}_{+} \times S \rho, S \rho=\left[x \in \mathbb{R}^{n}:\|x\|<\rho<\infty\right]$ and $f(\mathrm{t}, 0)=0$ for $t \geq 0$. In particular, $F(t, y)=f(t$, $y)+R(t, y)$, we have a special case of $(2.2 .3)$ and $R(t, y)$ is said to be perturbation term.

We will only give the basic existence and uniqueness result with the Lipschitz condition by using contraction mapping theorem and a weighted norm with Mittag-Leffler function in [4].

Theorem 2.2.1: Assume that

(i) $f \in C\left[R, \mathbb{R}^{n}\right]$ and bounded by $M$ on $R$ where $R=\left[(t, x): t_{0} \leq t \leq t_{0}+T,\left\|x-x_{0}\right\| \leq\right.$ $b]$

(ii) ||$f(t, x)-f(t, y)\|\leq L\| x-y \|, L>0,(t, x) \in R$ where the inequalities are componentwise.

Then there exist a unique solution $x(t)=x\left(t, t_{0}, x_{0}\right)$ on $\left[t_{0}, t_{0}+\alpha\right]$ for the initial value problem of the fractional order differential equation with Caputo's derivative of

(2.2.1) where $\alpha=\min \left[T,\left(\frac{b \Gamma(q+1)}{M}\right)^{\frac{1}{q}}\right]$.

Proof of Theorem 2.3.1, please see in [4].

Corollary 2.2.1: Let $0<q<1$, and $f:\left(t_{0}, t_{0}+T\right] \times S \rho \rightarrow \mathbb{R}$ be a function such that $f$ $(t, x) \in L\left(t_{0}, t_{0}+T\right)$ for any $x \in$.S $\rho$. If $x(t) \in L\left(t_{0}, t_{0}+T\right)$, then $x(t)$ satisfies a.e. the initial value problems of the fractional order differential equations with Caputo's 
derivative (2.2.5) if, and only if, $x(t)$ satisfies a.e. the Volterra fractional order integral equation (2.2.6).

Proof of Corollary 2.2.1, please see in [7].

We assume that we have sufficient conditions to the existence and uniqueness of solutions through $\left(t_{0}, x_{0}\right)$ and $\left(\tau_{0}, y_{0}\right)$. If $f \in C\left[\left[t_{0}, t_{0}+T\right] \times \mathbb{R}^{n}, \mathbb{R}^{n}\right]$ and $x(t) \in C^{q}\left[\left[t_{0}\right.\right.$, $T], \mathbb{R}]$ is the solution of

$$
{ }^{c} D^{q} x=f(t, x), x\left(t_{0}\right)=x_{0} \text { for } t \geq t_{0}, t_{0} \in \mathbb{R}_{+}
$$

where ${ }^{c} D^{q} x$ is the Caputo fractional order derivative of $x$ as in (2.1.1), then it also satisfies the Volterra fractional order integral equation

$$
x(t)=x_{0}+\frac{1}{\Gamma(q)} \int_{t_{0}}^{t}(t-s)^{q-1} f(s, x(s)) d s, \quad t_{0} \leq t \leq t_{0}+T
$$

and that every solution of (2.2.6) is also a solution of (2.2.5), for detail please see [7].

\subsection{ITD boundedness and Lagrange stability, fundamental Lemmata and Lyapunov-like function}

Before we establish our comparison theorem and boundedness criteria and Lagrange stability for initial time difference, we need to introduce the following definitions of ITD boundedness and Lagrange stability and Lyapunov-like functions.

Definition 2.3.1: The solution $y\left(t, \tau_{0}, y_{0}\right)$ of the initial value problems of fractional order differential equation with Caputo's derivative of (2.2.3) through $\left(\tau_{0}, y_{0}\right)$ is said to be initial time difference equi-bounded with respect to the solution $\tilde{x}\left(t, \tau_{0}, x_{0}\right)=x\left(t-\eta, t_{0}, x_{0}\right)$, where $x\left(t, t_{0}, x_{0}\right)$ is any solution of the initial value problems of fractional order differential equation with Caputo's derivative of (2.2.1) for $t$ $\geq \tau_{0}, \tau_{0} \in \mathbb{R}_{+}$and $\eta=\tau_{0}-t_{0}$ if and only if for any $\alpha>0$ there exist positive functions $\beta=\beta\left(\tau_{0}, \alpha\right)$ and $\gamma=\gamma\left(\tau_{0}, \alpha\right)$ that is continuous in $\tau_{0}$ for each $\alpha$ such that

$$
\left\|y_{0}-x_{0}\right\| \leq \alpha \text { and }\left|\tau_{0}-t_{0}\right| \leq \gamma \text { implies } \mid y\left(t, \tau_{0}, y_{0}\right)-x\left(t-\eta, t_{0}, x_{0}\right) \|<\beta \text { for } t \geq \tau_{0} .
$$

If $\beta$ and $\gamma$ are independent of $\tau_{0}$, then the solution $y\left(t, \tau_{0}, y_{0}\right)$ of the initial value problems of fractional order differential equation with Caputo's derivative of (2.2.3) is initial time difference uniformly equi-bounded with respect to the solution $x\left(t-\eta, t_{0}\right.$, $\left.x_{0}\right)$.

Definition 2.3.2: The solution $y\left(t, \tau_{0}, y_{0}\right)$ of the initial value problems of fractional order differential equation with Caputo's derivative of (2.2.3) through $\left(\tau_{0}, y_{0}\right)$ is said to be initial time difference quasi-equi-asymptotically stable (equi-attractive in the large) with respect to the solution $\tilde{x}\left(t, \tau_{0}, x_{0}\right)=x\left(t-\eta, t_{0}, x_{0}\right)$ for $t \geq \tau_{0}, \tau_{0} \in \mathbb{R}_{+}$if for each $\epsilon$ $>0$ and each $\alpha>0$ there exist a positive function $\gamma=\gamma\left(\tau_{0}, \alpha\right)$ and $T=T\left(\tau_{0}, \epsilon, \alpha\right)>0$ a number such that

$$
\left\|y_{0}-x_{0}\right\| \leq \alpha \text { and }\left|\tau_{0}-\tau_{0}\right| \leq \gamma \text { implies }\left\|y\left(t, \tau_{0}, y_{0}\right)-x\left(t-\eta, t_{0}, x_{0}\right)\right\|<\varepsilon \text { for } t \geq \tau_{0}+T .
$$

If $T$ and $\gamma$ are independent of $\tau_{0}$, then the solution $y\left(t, \tau_{0}, y_{0}\right)$ of the initial value problems of fractional order differential equation with Caputo's derivative of (2.2.3) is initial time difference uniformly quasi-equi-asymptotically stable with respect to the solution $x\left(t-\eta, t_{0}, x_{0}\right)$. If the Definition 2.3.1 and the Definition 2.3.2 hold together, then we have initial time difference Lagrange stability. 
Definition 2.3.3: A function $\varphi(r)$ is said to belong to the class $\mathcal{K}$ if $\varphi \in C\left[(0, \rho), \mathbb{R}_{+}\right]$, $\varphi(0)=0$, and $\varphi(r)$ is strictly monotone increasing in $r$. It is said to belong to class $\mathcal{K}_{\infty}$ if $\rho=\infty$ and $\varphi(r) \rightarrow \infty$ as $r \rightarrow \infty$.

Definition 2.3.4: For any Lyapunov-like function $V(t, x) \in C\left[\mathbb{R}_{+} \times \mathbb{R}^{n}, \mathbb{R}_{+}\right]$we define the fractional order Dini-derivatives in Caputo's sense ${ }^{c} D_{+}^{q} V(t, x)$ and ${ }^{c} D_{-}^{q} V(t, x)$ as follows

$$
\begin{aligned}
& { }^{c} D_{+}^{q} V(t, x)=\lim _{h \rightarrow 0^{+}} \sup \frac{1}{h^{q}}\left[V(t, x)-V\left(t-h, x-h^{q} f(t, x)\right)\right] \\
& { }^{c} D_{-}^{q} V(t, x)=\lim _{h \rightarrow 0^{-}} \inf \frac{1}{h^{q}}\left[V(t, x)-V\left(t-h, x-h^{q} f(t, x)\right)\right]
\end{aligned}
$$

for $(t, x) \in \mathbb{R}_{+} \times \mathbb{R}^{n}$.

Definition 2.3.5: For a real-valued function $V(t, x) \in C\left[\mathbb{R}_{+} \times \mathbb{R}^{n}, \mathbb{R}_{+}\right]$we define the generalized fractional order derivatives (Dini-like derivatives) in Caputo's sense ${ }_{*}^{c} D_{+}^{q} V(t, y-\tilde{x})$ and ${ }_{*}^{c} D_{-}^{q} V(t, y-\tilde{x})$ as follows

$$
\begin{aligned}
& { }_{*}^{c} D_{+}^{q} V\left(t, y-\tilde{x}=\lim _{h \rightarrow 0^{+}} \sup \frac{1}{h^{q}}\left[V(t, y-\tilde{x})-V\left(t-h, y-\tilde{x}-h^{q}(F(t, y)-\tilde{f}(t, \tilde{x}))\right)\right]\right. \\
& { }_{*}^{c} D_{-}^{q} V\left(t, y-\tilde{x}=\lim _{h \rightarrow 0^{-}} \inf \frac{1}{h^{q}}\left[V(t, y-\tilde{x})-V\left(t-h, y-\tilde{x}-h^{q}(F(t, y)-\tilde{f}(t, \tilde{x}))\right)\right]\right.
\end{aligned}
$$

for $(t, x) \in \mathbb{R}_{+} \times \mathbb{R}^{n}$.

Lemma 2.3.1: (see [14]) Let $f, F \in C\left[\left[t_{0}, T\right] \times \mathbb{R}^{n}, \mathbb{R}^{n}\right]$, and let

$$
G(t, r)=\max _{\tilde{x}, y \in \bar{B}\left(x_{0} ; r\right)}\|F(t, y)-\tilde{f}(t, \tilde{x})\|
$$

where $G(t, r) \in C\left[\mathbb{R}_{+} \times \mathbb{R}_{+}, \mathbb{R}_{+}\right]$and $\bar{B}$ is closed ball with center at $x_{0}$ and radius $r$. Assume that $r^{*}\left(t, \tau_{0},\left\|y_{0}-x_{0}\right\|\right)$ is the maximal solution of initial value problem of fractional order differential equation with Caputo' s derivative $\mathrm{d}^{c} D^{q} u=G(t, u), u\left(\tau_{0}\right)$ $=\left\|y_{0}-x_{0}\right\|$ through $\left(\tau_{0},\left\|y_{0}-x_{0}\right\|\right) . \tilde{x}\left(t, \tau_{0}, x_{0}\right)=x\left(t-\eta, t_{0}, x_{0}\right)$ and $y\left(t, \tau_{0}, y_{0}\right)$ is the solution of fractional order differential equation (2.2.3) with Caputo's derivatives. Then

$$
\left\|y\left(t, \tau_{0}, y_{0}\right)-x\left(t-\eta, t_{0}, x_{0}\right)\right\| \leq r^{*}\left(t, \tau_{0},\left\|y_{0}-x_{0}\right\|\right) \text { for } t \geq \tau_{0} .
$$

Lemma 2.3.2: (see [14]) Let $V(t, z) \in C\left[\mathbb{R}_{+} \times \mathbb{R}^{n}, \mathbb{R}_{+}\right]$and $V(t, z)$ be locally Lipschitzian in $z$. Assume that the generalized fractional order derivatives (Dini-like derivatives) in Caputo's sense

$$
{ }_{*}^{c} D_{+}^{q} V(t, y-\tilde{x})=\lim _{h \rightarrow 0^{+}} \sup \frac{1}{h^{q}}\left[V(t, y-\tilde{x})-V\left(t-h, y-\tilde{x}-h^{q}(F(t, y)-\bar{f}(t, \tilde{x}))\right)\right]
$$

satisfies ${ }_{*}^{c} D_{+}^{q} V(t, y-\tilde{x}) \leq G(t, V(t, y-\tilde{x}))$ with $(t, \tilde{x}),(t, y) \in \mathbb{R}_{+} \times \mathbb{R}^{n}$, where $G(t, u) \in$ $C\left[\mathbb{R}_{+} \times \mathbb{R}_{+}, \mathbb{R}\right]$. Let $r(t)=r\left(t, \tau_{0}, u_{0}\right)$ be the maximal solution of the fractional order differential equation ${ }^{c} D^{q} u=G(t, u), u\left(\tau_{0}\right)=u_{0} \geq 0$, for $t \geq t_{0}$. If $\tilde{x}(t)=x\left(t-\eta, t_{0}, x_{0}\right)$ and $y$ $(t)=y\left(t, \tau_{0}, y_{0}\right)$ is any solution of (2.2.3) for $t \geq \tau_{0}$ such that $V\left(\tau_{0}, y_{0}-x_{0}\right) \leq u_{0}$ then $V(t, y(t)-\tilde{x}(t)) \leq r(t)$ for $t \geq \tau_{0}$. 


\section{Initial time difference fractional comparison results}

In this section, we have an other comparison result in which the boundedness and Lagrange stability properties of the null solution of the comparison system imply the corresponding initial time difference boundedness and Lagrange stability properties of the perturbed fractional order differential system in Caputo's sense with respect to the unperturbed fractional order differential system in Caputo' s sense.

\subsection{ITD boundedness criteria and Lagrange stability}

Theorem 3.1.1: Assume that

(i) Let $V(t, z) \in C\left[\mathbb{R}_{+} \times \mathbb{R}^{n}, \mathbb{R}_{+}\right]$be locally Lipschitzian in $z$ and the fractional order Dini derivatives in Caputo's sense ${ }^{c} D_{+}^{q} V(t, \gamma(t)-\tilde{x}(t))$

$$
{ }^{c} D_{+}^{q} V(t, \gamma(t)-\tilde{x}(t)) \leq \lim _{h \rightarrow 0^{+}} \sup \frac{1}{h^{q}}\left[V(t, \gamma(t)-\tilde{x}(t))-V\left(t-h,(\gamma-\tilde{x})-h^{q}(F(t, y)-\tilde{f}(t, \tilde{x}))\right)\right]
$$

satisfies ${ }_{*}^{c} D_{+}^{q} V(t, y-\tilde{x}) \leq G(t, V(t, y-\tilde{x}))$ for $(t, \tilde{x}),(t, y) \in \mathbb{R}_{+} \times \mathbb{R}^{n},(t, y) \in \mathbb{R}_{+} \times \mathbb{R}^{n}$, where $G(t, u) \in C\left[\mathbb{R}_{+} \times \mathbb{R}_{+}, \mathbb{R}\right]$ and the generalized fractional order (Dini-like) derivatives in Caputo's sense ${ }_{*}^{c} D_{+}^{q} V(t, x)$;

(ii) Let $V(t, x)$ be positive definite such that

$$
b(\| x||) \leq V(t, x) \text { with }(t, x) \in \mathbb{R}_{+} \times \mathbb{R}^{n}
$$

and $b \in \mathcal{K}, b(u) \rightarrow \infty$ as $u \rightarrow \infty$ on the interval $0 \leq u<\infty$;

(iii) Let $r(t)=r\left(t, \tau_{0}, u_{0}\right)$ be the maximal solution of the fractional order differential equation with Caputo's derivative

$$
{ }^{c} D^{q} u=G(t, u), \quad u\left(\tau_{0}\right)=u_{0} \geq 0 \text { for } t \geq \tau_{0} .
$$

Then the boundedness properties of the null solution of the fractional order differential system with Caputo's derivative (3.1.2) with $G(t, 0)=0$ imply the corresponding initial time difference boundedness properties of $y\left(t, \tau_{0}, y_{0}\right)$ any solution of fractional order differential system with Caputo's derivative (2.2.3) with respect to $\tilde{x}\left(t, \tau_{0}, x_{0}\right)=x\left(t-\eta, t_{0}, x_{0}\right)$ where $x\left(t, t_{0}, x_{0}\right)$ is any solution of fractional order differential system with Caputo's derivative of (2.2.1).

Proof: Let $\alpha>0$ and $\tau_{0} \in \mathbb{R}_{+}$be given, and let ||$y_{0}-x_{0}||<\alpha$ and $\left|\tau_{0}-t_{0}\right| \leq \gamma$ for $\gamma$ $\left(\tau_{0}, \alpha\right)>0$. In view of the hypotheses on $V(t, x)$, there exists a number $\alpha_{1}=\alpha_{1}\left(\tau_{0}, \alpha\right)>$ 0 satisfying the inequalities

$$
|| y_{0}-x_{0}|| \leq \alpha \text { and }\left|\tau_{0}-t_{0}\right| \leq \gamma, V\left(\tau_{0}, y_{0}-x_{0}\right) \leq \alpha_{1}
$$

together. Assume that comparison system (3.1.2) is equi-bounded. Then, given $\alpha_{1} \geq$ 0 and $\tau_{0} \in \mathbb{R}_{+}$there exist a $\beta_{1}=\beta_{1}\left(\tau_{0}, \alpha\right)$ that is continues in $\tau_{0}$ for each $\alpha$ such that

$$
r\left(t, \tau_{0}, u_{0}\right)<\beta_{1} \text { provided } u_{0} \leq \alpha_{1} .
$$

Moreover, $b(u) \rightarrow \infty$ as $u \rightarrow \infty$, we can choose a $L=L\left(\tau_{0}, \alpha\right)$ verifying the relation

$$
b(L) \geq \beta_{1}\left(\tau_{0}, \alpha\right) .
$$

Now let $u_{0}=V\left(\tau_{0}, y_{0}-x_{0}\right)$. Then assumption $(i)$ and Lemma 2.3.2 show that

$$
V\left(t, y\left(t, \tau_{0}, y_{0}\right)-x\left(t-\eta, t_{0}, x_{0}\right)\right) \leq r\left(t, \tau_{0}, u_{0}\right) \text { for } t \geq \tau_{0}
$$


where $r\left(t, \tau_{0}, u_{0}\right)$ is the maximal solution of comparison equation (3.1.2). Suppose, if possible, that there is a solution of system (2.2.4) $w\left(t, \tau_{0}, w_{0}\right)=y\left(t, \tau_{0}, y_{0}\right)-x\left(\mathrm{t}-\eta, t_{0}\right.$, $x_{0}$ ) for $t \geq \tau_{0}$ with $\left\|y_{0}-x_{0}\right\|<\alpha$ having the property that, for some $t_{1}>\tau_{0}$, $t_{1}>\tau_{0}, \| \gamma\left(t, \tau_{0}, y_{0}\right)-\tilde{x}\left(t, \tau_{0}, x_{0} \|=L\right.$. Then because of relations (3.1.1), (3.1.3), (3.1.4) and (3.1.5), there results oddity

$$
b(L) \leq V\left(t_{1}, \gamma\left(t_{1}, \gamma\left(t_{1}, \tau_{0}, y_{0}\right)-x\left(t_{1}, \tau_{0}, x_{0}\right)\right) \leq r\left(t_{1}, \tau_{0}, u_{0}\right)<\beta_{1}\left(\tau_{0}, \alpha\right) \leq b(L)\right.
$$

then

$$
\left\|\gamma\left(t, \tau_{0}, y_{0}\right)-\tilde{x}\left(t, \tau_{0}, x_{0}\right)\right\|<L\left(\tau_{0}, \alpha\right) \text { provided }\left\|y_{0}-x_{0}\right\| \leq \alpha .
$$

These complete the proof.

Theorem 3.1.2: Let the assumption of Theorem 3.1.1 holds. Then the quasi-equiasymptotically stability properties of the null solution of the fractional order differential system with Caputo's derivative (3.1.2) with $G(t, 0)=0$ imply the corresponding initial time difference quasi-equi-asymptotically stability properties of $y\left(t, \tau_{0}, y_{0}\right)$ any solution of fractional order differential system with Caputo's derivative (2.2.3) with respect to $\tilde{x}\left(t, \tau_{0}, x_{0}\right)=x\left(t-\eta, t_{0}, x_{0}\right)$ where $x\left(t, t_{0}, x_{0}\right)$ is any solution of fractional order differential system with Caputo's derivative of (2.2.1).

Proof: We want to prove the theorem by considering Definition 2.3.2.

Let $\epsilon>0, \alpha \geq 0$ and $\tau_{0} \in \mathbb{R}_{+}$be given and let ||$y_{0}-x_{0} \| \leq \alpha$ and $\left|\tau_{0}-t_{0}\right| \leq \gamma$ for $\gamma\left(\tau_{0}\right.$, $\alpha)>0$. As in the proof of the Theorem 3.1.1, there exists a $\alpha_{1}=\alpha_{1}\left(\tau_{0}, \alpha\right)$ satisfying

$$
|| y_{0}-x_{0}|| \leq \alpha \text { and }\left|\tau_{0}-t_{0}\right| \leq \gamma, V\left(\tau_{0}, y_{0}-x_{0}\right) \leq \alpha_{1}
$$

simultaneously. Since for comparison system (3.1.2) is quasi-equi-asymptotically stable. Then, given $\alpha_{1} \geq 0, b(\epsilon)$ and $\tau_{0} \in \mathbb{R}_{+}$there exist a $T=T\left(\tau_{0}, \alpha, \epsilon\right)$ such that

$$
u_{0} \leq \alpha_{1} \text { implies } r\left(t, \tau_{0}, u_{0}\right)<b(\varepsilon) \text { for } t \geq \tau_{0}+T \text {. }
$$

Choose $u_{0}=V\left(\tau_{0}, y_{0}-x_{0}\right)$. Then assumption $(i)$ and Lemma 2.3.2 show that

$$
V\left(t, y\left(t, \tau_{0}, y_{0}\right)-x\left(t-\eta, t_{0}, x_{0}\right)\right) \leq r\left(t, \tau_{0}, u_{0}\right), t \geq \tau_{0}
$$

If possible, let there exist a sequence $\left\{t_{k}\right\}$,

$$
t_{k} \geq \tau_{0}+T, t_{k} \rightarrow \infty \text { as } k \rightarrow \infty
$$

such that, for some solution of system (2.2.4) $w\left(t, \tau_{0}, w_{0}\right)=y\left(t, \tau_{0}, y_{0}\right)-x\left(t-\eta, t_{0}, x_{0}\right)$ for $t \geq \tau_{0}$ with $\left\|y_{0}-x_{0}\right\| \leq \alpha$ we have

$$
\left\|\gamma\left(t, \tau_{0}, y_{0}\right)-\tilde{x}\left(t, \tau_{0}, x_{0}\right)\right\| \geq \varepsilon
$$

This implies, in view of the inequalities (3.1.1), (3.1.6) and (3.1.7)

$$
b(\varepsilon) \leq V\left(t_{k}, y\left(t_{k}, \tau_{0}, y_{0}\right)-x\left(t_{k}, \tau_{0}, x_{0}\right)\right) \leq r\left(t_{k}, \tau_{0}, u_{0}\right)<b(\varepsilon)
$$

which proves

$$
\left\|\gamma\left(t, \tau_{0}, y_{0}\right)-\tilde{x}\left(t, \tau_{0}, x_{0}\right)\right\|<\varepsilon \text { provided }\left\|y_{0}-x_{0}\right\| \leq \alpha \text { for } t \geq \tau_{0}+T\left(\tau_{0}, \varepsilon, \alpha\right) .
$$

Therefore, these complete the proof.

Theorem 3.1.3: Let the assumption of Theorem 3.1.1 holds as

(i) Let $V(t, z) \in C\left[\mathbb{R}_{+} \times \mathbb{R}^{n}, \mathbb{R}_{+}\right]$be locally Lipschitzian in $z$ and the fractional order Dini derivatives in Caputo' s sense ${ }^{c} D_{+}^{q} V(t, y(t)-\tilde{x}(t))$ 


$$
{ }^{c} D_{+}^{q} V(t, y(t)-\tilde{x}(t)) \leq \lim _{h \rightarrow 0^{+}} \sup \frac{1}{h^{q}}\left[V(t, y(t)-\tilde{x}(t))-V\left(t-h,(\gamma-\tilde{x})-h^{q}(F(t, y)-\tilde{f}(t, \tilde{x}))\right)\right]
$$

satisfies ${ }_{*}^{c} D_{+}^{q} V(t, y-\tilde{x}) \leq G(t, V(t, y-\tilde{x}))$ for $(t, \tilde{x}),(t, y) \in \mathbb{R}_{+} \times \mathbb{R}^{n},(t, y) \in \mathbb{R}_{+} \times \mathbb{R}^{n}$, where $G(t, u) \in C\left[\mathbb{R}_{+} \times \mathbb{R}_{+}, \mathbb{R}\right]$ and the generalized fractional order (Dini-like) derivatives in Caputo's sense ${ }_{*}^{c} D_{+}^{q} V(t, x)$;

(ii) Let $V(t, x)$ be positive definite such that

$$
b(\| x||) \leq V(t, x) \text { with }(t, x) \in \mathbb{R}_{+} \times \mathbb{R}^{n}
$$

and $b \in \mathcal{K}, b(u) \rightarrow \infty$ as $u \rightarrow \infty$ on the interval $[0, \infty)$;

(iii) Let $r(t)=r\left(t, \tau_{0}, u_{0}\right)$ be the maximal solution of the fractional order differential equation with Caputo's derivative

$$
{ }^{c} D^{q} u=G(t, u), \quad u\left(\tau_{0}\right)=u_{0} \geq 0 \text { for } t \geq \tau_{0} .
$$

Then the boundedness and Lagrange stability properties of the null solution of the fractional order differential system with Caputo' s derivative $(3.1 .2)$ with $G(t, 0)=0$ imply the corresponding initial time difference boundedness and Lagrange stability properties of $y\left(t, \tau_{0}, y_{0}\right)$ any solution of fractional order differential system with Caputo's derivative (2.2.3) with respect to $\tilde{x}\left(t, \tau_{0}, x_{0}\right)=x\left(t-\eta, t_{0}, x_{0}\right)$ where $x\left(t, t_{0}, x_{0}\right)$ is any solution of fractional order differential system with Caputo's derivative of (2.2.1).

Proof: We know that equi-Lagrange stability requires the equi-boundedness and quasi-equi-asymptotically stability. We proved in Theorem 3.1.1 and Theorem 3.1.2, respectively. Then the proof of Theorem 3.1.3 is complete.

\subsection{ITD uniformly boundedness criteria and Lagrange stability}

Theorem 3.2.1: Assume that the assumptions of Theorem 3.1.1 hold. In addition to hypotheses of Theorem 3.1.1, let $V(t, x)$ verify the inequality

$$
V(t, x) \leq a(\|x\|) \text { with }(t, x) \in \mathbb{R}_{+} \times \mathbb{R}^{n}
$$

where $a \in \mathcal{K}$ on the interval $[0, \infty)$.

Then, if fractional order comparison system (3.1.2) is uniformly bounded, the solution $y\left(t, \tau_{0}, y_{0}\right)$ of (2.2.3) through $\left(\tau_{0}, y_{0}\right)$ is initial time difference uniformly bounded for $t \geq \tau_{0} \in \mathbb{R}_{+}$with respect to the solution $x\left(t-\eta, t_{0}, x_{0}\right)$ through $\left(t_{0}, x_{0}\right)$ where $x\left(t, t_{0}\right.$, $\left.x_{0}\right)$ is the solution of (2.2.1) through $\left(t_{0}, x_{0}\right)$.

Proof: Let $\alpha \geq 0$ and $\tau_{0} \in \mathbb{R}_{+}$be given, and let ||$y_{0}-x_{0} \| \leq \alpha,\left|\tau_{0}-t_{0}\right| \leq \gamma$ for $\gamma(\alpha)>$ 0 . In view of the hypotheses on $V(t, x)$, there exists a number $\alpha_{1}=a(\alpha)$ satisfying the inequalities

$$
\left\|y_{0}-x_{0}\right\| \leq \alpha, \quad V\left(\tau_{0}, y_{0}-x_{0}\right) \leq \alpha_{1}=a(\alpha)
$$

together. Assume that fractional order comparison system (3.1.2) is uniformly equibounded. Then, given $\alpha_{1} \geq 0$ and $\tau_{0} \in \mathbb{R}_{+}$there exist a $\beta_{1}=\beta_{1}(\alpha)$ such that

$$
r\left(t, \tau_{0}, u_{0}\right)<\beta_{1} \text { provided } u_{0} \leq \alpha_{1}\left(\beta_{1} \text { and } \alpha_{1} \text { are independent of } \tau_{0}\right) .
$$

Moreover, $b(u) \rightarrow \infty$ as $u \rightarrow \infty$, we can choose a $L=L(\alpha)$ verifying the relation

$$
b(L) \geq \beta_{1}(\alpha)
$$


Now let $u_{0}=V\left(\tau_{0}, y_{0}-x_{0}\right)$. Then assumption $(i)$ and Lemma 2.3.2 show that

$$
V\left(t, y\left(t, \tau_{0}, y_{0}\right)-x\left(t-\eta, t_{0}, x_{0}\right)\right) \leq r\left(t, \tau_{0}, u_{0}\right), t \geq \tau_{0}
$$

where $r\left(t, \tau_{0}, u_{0}\right)$ is the maximal solution of comparison equation (3.1.2). Suppose, if possible, that there is a solution of system (2.2.4) $w\left(t, \tau_{0}, w_{0}\right)=y\left(t, \tau_{0}, y_{0}\right)-x\left(t-\eta, t_{0}\right.$, $x_{0}$ ) for $t \geq \tau_{0}$ with $\left\|y_{0}-x_{0}\right\| \leq \alpha$ having the property that, for some $t_{1}>\tau_{0}$,

$$
\left\|y\left(t_{1}, \tau_{0}, y_{0}\right)-\tilde{x}\left(t_{1}, \tau_{0}, x_{0}\right)\right\|=L
$$

where $L$ is independent of $\tau_{0}$. Then because of relations (3.1.1), (3.1.5), (3.2.2) and (3.2.3), there results contradiction

$$
b(L) \leq V\left(t_{1}, \gamma\left(t_{1}, \tau_{0}, y_{0}\right)-\tilde{x}\left(t_{1}, \tau_{0}, x_{0}\right)\right) \leq r\left(t_{1}, \tau_{0}, u_{0}\right)<\beta_{1}(\alpha) \leq b(L) .
$$

Therefore,

$$
\left\|y\left(t, \tau_{0}, y_{0}\right)-\tilde{x}\left(t, \tau_{0}, x_{0}\right)\right\|<L(\alpha) \text { provided }\left\|y_{0}-x_{0}\right\| \leq \alpha \text { and }\left|\tau_{0}-t_{0}\right| \leq \gamma \text { for } \gamma(\alpha)>0 \text { and } t \geq \tau_{0} .
$$

These completes the proof.

Theorem 3.2.2: Assume that the assumptions of Theorem 3.2.1 holds. Then, if fractional order comparison system (3.1.2) is uniformly quasi-equi-asymptotically stable, the solution $y\left(t, \tau_{0}, y_{0}\right)$ of (2.2.3) through $\left(\tau_{0}, y_{0}\right)$ is initial time difference uniformly quasi-equi-asymptotically stable for $t \geq \tau_{0} \in \mathbb{R}_{+}$with respect to the solution $x\left(t-\eta, t_{0}\right.$, $\left.x_{0}\right)$ through $\left(t_{0}, x_{0}\right)$ where $x\left(t, t_{0}, x_{0}\right)$ is the solution of (2.2.1) through $\left(t_{0}, x_{0}\right)$.

Proof: We want to prove the theorem by considering Definition 2.3.2 as independent of $\tau_{0}$. Let $\epsilon>0, \alpha \geq 0$ and $\tau_{0} \in \mathbb{R}_{+}$be given. and let ||$y_{0}-x_{0} \| \leq \alpha$ and $\left|\tau_{0}-t_{0}\right| \leq \gamma$ for $\gamma(\alpha)>0$.

As in the preceding proof, there exists a $\alpha_{1}=a(\alpha)$ satisfying

$$
\left\|y_{0}-x_{0}\right\| \leq \alpha, V\left(\tau_{0}, y_{0}-x_{0}\right) \leq \alpha_{1}
$$

simultaneously. Since for comparison system (3.1.2) is uniformly quasi- equi-asymptotically stable. Then, given $\alpha_{1} \geq 0, b(\epsilon)>0$ and $\tau_{0} \in \mathbb{R}_{+}$there exist a $T=T(\alpha, \epsilon)$ such that

$$
u_{0} \leq \alpha_{1} \text { implies } r\left(t, \tau_{0}, u_{0}\right)<b(\epsilon) \text { for } t \geq \tau_{0}+T
$$

Choose $u_{0}=V\left(\tau_{0}, y_{0}-x_{0}\right)$. Then assumption $(i)$ and Lemma 2.3.2 show that

$$
V\left(t, y\left(t, \tau_{0}, y_{0}\right)-x\left(t-\eta, t_{0}, x_{0}\right)\right) \leq r\left(t, \tau_{0}, u_{0}\right), t \geq \tau_{0}
$$

If possible, let there exist a sequence $\left\{t_{k}\right\}$,

$$
t_{k} \geq \tau_{0}+T, t_{k} \rightarrow \infty \text { as } k \rightarrow \infty
$$

such that, for some solution of system (2.2.4) $w\left(t, \tau_{0}, w_{0}\right)=y\left(t, \tau_{0}, y_{0}\right)-x\left(t-\eta, t_{0}, x_{0}\right)$ for $t \geq \tau_{0}$ with $\left\|y_{0}-x_{0}\right\| \leq \alpha$ we have

$$
\left\|\gamma\left(t, \tau_{0}, y_{0}\right)-\tilde{x}\left(t, \tau_{0}, x_{0}\right)\right\| \geq \varepsilon
$$

This implies, in view of the inequalities (3.1.1), (3.1.5) and (3.2.4), we obtain

$$
b(\varepsilon) \leq V\left(t_{k}, y\left(t_{k}, \tau_{0}, y_{0}\right)-x\left(t_{k}, \tau_{0}, x_{0}\right)\right) \leq r\left(t_{k}, \tau_{0}, u_{0}\right)<b(\varepsilon)
$$


which proves

$$
\left\|y\left(t, \tau_{0}, \gamma_{0}\right)-\tilde{x}\left(t, \tau_{0}, x_{0}\right)\right\|<\varepsilon \text { provided }\left\|y_{0}-x_{0}\right\| \leq \alpha \text { and }\left|\tau_{0}-t_{0}\right| \leq \gamma \text { for } \gamma(\alpha)>0 \text { and } t \geq \tau_{0}+T(\varepsilon,
$$

Therefore, these complete the proof.

Theorem 3.2.3: Assume that

(i) Let $V(t, z) \in C\left[\mathbb{R}_{+} \times \mathbb{R}^{n}, \mathbb{R}_{+}\right]$be locally Lipschitzian in $z$ and the fractional order Dini derivatives in Caputo's sense ${ }^{c} D_{+}^{q} V(t, y(t)-\tilde{x}(t))$

$$
{ }^{c} D_{+}^{q} V(t, y(t)-\tilde{x}(t)) \leq \lim _{h \rightarrow 0^{+}} \sup \frac{1}{h^{q}}\left[V(t, y(t)-\tilde{x}(t))-V\left(t-h,(\gamma-\tilde{x})-h^{q}(F(t, y)-\tilde{f}(t, \tilde{x}))\right)\right]
$$

satisfies ${ }_{*}^{c} D_{+}^{q} V(t, y-\tilde{x}) \leq G(t, V(t, y-\tilde{x}))$ for $(t, \tilde{x}),(t, y) \in \mathbb{R}_{+} \times \mathbb{R}^{n}$, where $G(t, u) \in$ $C\left[\mathbb{R}_{+} \times \mathbb{R}_{+}, \mathbb{R}\right]$ and the generalized fractional order (Dini-like) derivatives in Caputo's sense ${ }_{*}^{c} D_{+}^{q} V(t, x)$;

(ii) Let $V(t, x)$ be positive definite such that

$$
b(\|x\|) \leq V(t, x) \leq a(\|x\|) \text { with }(t, x) \in \mathbb{R}_{+} \times \mathbb{R}^{n}
$$

and $a, b \in \mathcal{K}, b(u) \rightarrow \infty$ as $u \rightarrow \infty$ on the interval $0 \leq u<\infty$;

(iii) Let $r(t)=r\left(t, \tau_{0}, u_{0}\right)$ be the maximal solution of the fractional order differential equation

with Caputo's derivative

$$
{ }^{c} D^{q} u=G(t, u), \quad u\left(\tau_{0}\right)=u_{0} \geq 0 \text { for } t \geq \tau_{0} .
$$

Then the uniform-Lagrange stability properties of the null solution of the fractional order differential system with Caputo's derivative (3.1.2) with $G(t, 0)=0$ imply the corresponding initial time difference uniform-Lagrange stability properties of $y\left(t, \tau_{0}, y_{0}\right)$ any solution of fractional order differential system with Caputo's derivative (2.2.3) with respect to $\tilde{x}\left(t, \tau_{0}, x_{0}\right)=x\left(t-\eta, t_{0}, x_{0}\right)$ where $x\left(t, t_{0}, x_{0}\right)$ is any solution of fractional order differential system with Caputo's derivative of (2.2.1).

Proof: By using the Theorem 3.2.1 and the Theorem 3.2.2, we have that the uniform bounded-ness and uniformly quasi-equi-asymptotically stability properties of comparison system (3.1.2) imply the corresponding uniform boundedness and uniformly quasiequi-asymptotically stability properties of $y\left(t, \tau_{0}, y_{0}\right)$ of perturbed differential system of (2.2.3) that differs in initial position and initial time with respect to the solution $x(t$ $\left.\eta, t_{0}, x_{0}\right)$, where $x\left(t, t_{0}, x_{0}\right)$ is any solution of the unperturbed differential system of (2.2.1). These completes the proof.

\section{An example}

Example 4.1: Let us consider the unperturbed nonlinear fractional order vector differential system with the order $q$ in Caputo's sense for $t \geq \tau_{0}, \tau_{0} \in \mathbb{R}$ as follows

$$
\begin{aligned}
& { }^{c} D^{q} \tilde{x}=\left[\begin{array}{l}
{ }^{c} D^{q} \tilde{x}_{1} \\
{ }^{c} D^{q} \tilde{x}_{2}
\end{array}\right]=\left[\begin{array}{c}
-\tilde{x}_{1}+2 \tilde{x}_{2} y_{2}+2 y_{1} \\
y_{2}+y_{1} y_{2}+\tilde{x}_{1} \tilde{x}_{2}
\end{array}\right]=\tilde{f}(t, \tilde{x}, y), t \in \mathbb{R} \\
& {\left[\begin{array}{c}
\tilde{x}_{1}\left(\tau_{0}\right) \\
\tilde{x}_{2}\left(\tau_{0}\right)
\end{array}\right]=\left[\begin{array}{c}
\tilde{x}_{01} \\
\tilde{x}_{02}
\end{array}\right]=\tilde{x} \text { for } t \geq \tau_{0}, \tau_{0} \in \mathbb{R} \text { where }\left[\begin{array}{l}
\tilde{x}_{1}\left(\tau_{0}\right) \\
\tilde{x}_{2}\left(\tau_{0}\right)
\end{array}\right]=\left[\begin{array}{l}
\tilde{x}_{1}\left(\tau_{0}, \tau_{0}, x_{0}\right) \\
\tilde{x}_{2}\left(\tau_{0}, \tau_{0}, x_{0}\right)
\end{array}\right]}
\end{aligned}
$$

and its perturbed nonlinear fractional order vector differential system of (4.1 (a)) with the order $q$ in Caputo's sense for $t \geq \tau_{0}, \tau_{0} \in \mathbb{R}$ as follows 


$$
\begin{aligned}
& { }^{c} D^{q} y=\left[\begin{array}{l}
{ }^{c} D^{q} y_{1} \\
{ }^{c} D^{q} y_{2}
\end{array}\right]=\left[\begin{array}{c}
y_{1}+y_{2}^{2}+\tilde{x}_{2}^{2} \\
\tilde{x}_{2}+y_{1} \tilde{x}_{2}+\tilde{x}_{1} y_{2}
\end{array}\right]=F(t, \tilde{x}, y), t \in \mathbb{R} \\
& {\left[\begin{array}{l}
y_{1}\left(\tau_{0}\right) \\
y_{2}\left(\tau_{0}\right)
\end{array}\right]=\left[\begin{array}{l}
y_{01} \\
y_{02}
\end{array}\right]=y_{0} \text { for } t \geq \tau_{0} \text { where }\left[\begin{array}{l}
y_{1}\left(\tau_{0}\right) \\
y_{2}\left(\tau_{0}\right)
\end{array}\right]=\left[\begin{array}{l}
y_{1}\left(\tau_{0}, \tau_{0}, x_{0}\right) \\
y_{2}\left(\tau_{0}, \tau_{0}, x_{0}\right)
\end{array}\right]}
\end{aligned}
$$

where the perturbation term $R(t, \tilde{x}, y)$ of $(4.2(\mathrm{a}))$ is

$$
R(t, \tilde{x}, y)=\left[\begin{array}{c}
\tilde{x}_{1}-y_{1}+y_{2}^{2}+\tilde{x}_{2}^{2}-2 \tilde{x}_{2} y_{2} \\
\tilde{x}_{2}-y_{2}+y_{1} \tilde{x}_{2}+\tilde{x}_{1} y_{2}-y_{1} y_{2}-\tilde{x}_{1} \tilde{x}_{2}
\end{array}\right] \text { for } t \geq \tau_{0 .}
$$

Let us choose the Lyapunov function as $V(t, y-\tilde{x})=|| \gamma-\tilde{x}||=\left|y_{1}-\tilde{x}_{1}\right|+\left|y_{2}-\tilde{x}_{2}\right|$ and $y-\tilde{x} \geq 0$ where $\|y-\tilde{x}\|$ is the norm defined by standard metric in componentwise.

Let $a, b \in K$ be defined by

$$
a(\|y-\tilde{x}\|)=2\|y-\tilde{x}\|, b(\|y-\tilde{x}\|)=\frac{1}{2}\|y-\tilde{x}\|
$$

so that we have

$$
b(\|y-\tilde{x}\|) \leq V(t, y-\tilde{x}) \leq a(\|y-\tilde{x}\|) .
$$

Thus, $V$ is positive definite and decrescent. The Dini-like derivative of $V(t, y-\tilde{x})$ by substituting in for ${ }^{c} D^{q} \tilde{x}_{1},{ }^{c} D^{q} \tilde{x}_{2},{ }^{c} D^{q} y_{1}$ and ${ }^{c} D^{q} y_{2}$ yields

$$
\begin{aligned}
{ }_{*}^{c} D_{+}^{q} V(t, y-\tilde{x}) & \left.=\lim _{h \rightarrow 0^{+}} \sup \frac{1}{h^{q}}\left[V(t, y-\tilde{x})-V\left(t-h, y-\tilde{x}-h^{q}(F(t, \tilde{x}, y)-\tilde{f}(t, \tilde{x}, y))\right)\right)\right] \\
& ={ }^{c} D^{q} y_{1}(t)+{ }^{c} D^{q} \gamma_{2}(t)-\left[{ }^{c} D^{q} \tilde{x}_{1}(t)+{ }^{c} D^{q} \tilde{x}_{2}(t)\right] \\
& =y_{1}+y_{2}^{2}+\tilde{x}_{2}^{2}+\tilde{x}_{2}+y_{1} \tilde{x}_{2}+\tilde{x}_{1} y_{2}+\tilde{x}_{1}-2 \tilde{x}_{2} y_{2}-2 y_{1}-y_{2}-y_{1} y_{2}-\tilde{x}_{1} \tilde{x}_{2} \\
& =-\left[\left(y_{2}-\tilde{x}_{2}\right)\left[y_{1}-\tilde{x}_{1}+\tilde{x}_{2}-y_{2}+1\right]+\left(y_{1}-\tilde{x}_{1}\right)\right] \\
& \leq-\alpha\left[\left|y_{1}-\tilde{x}_{1}\right|+\left|y_{2}-\tilde{x}_{2}\right|\right] \text { where } \alpha>0 \text { depends on the order } q . \\
{ }_{*}^{c} D_{+}^{q} V(t, y-\tilde{x}) & \leq-\alpha V(t, y-\tilde{x}) .
\end{aligned}
$$

And we have

$$
{ }_{*}^{c} D_{+}^{q} V(t, y-\tilde{x}) \leq-\alpha V(t, y-\tilde{x}) .
$$

We apply Theorem 3.2.3 with the comparison system

$$
{ }^{c} D^{q} u=-\alpha u, u\left(\tau_{0}\right)=\left\|y_{0}-\tilde{x}_{0}\right\| \geq 0
$$

for $t \geq \tau_{0}, \tau_{0} \in \mathbb{R}$.

Hence, the Lagrange stability properties of the comparison equation in $u$ imply that the corresponding initial time difference Lagrange stability properties of the $y\left(t, \tau_{0}, y_{0}\right)$ of the system (4.2) with respect to the solution $\tilde{x}\left(t, \tau_{0}, x_{0}\right)$ of the system (4.1) for $t \geq \tau_{0}$, $\tau_{0} \in \mathbb{R}$.

Remark 4.2: Let $q$ be the integer order 1 and the systems (4.1 (a)) and (4.2 (a)) become the nonlinear vector differential systems for $t \geq \tau_{0} \geq 0$ on $\mathbb{R}$

$$
\tilde{x}^{\prime}=\left[\begin{array}{c}
\tilde{x}_{1}^{\prime} \\
\tilde{x}_{2}^{\prime}
\end{array}\right]=\left[\begin{array}{c}
-\tilde{x}_{1}+2 \tilde{x}_{2} y_{2}+2 y_{1} \\
y_{2}+y_{1} y_{2}+\tilde{x}_{1} \tilde{x}_{2}
\end{array}\right], t \in \mathbb{R}
$$


and its perturbed system

$$
y^{\prime}=\left[\begin{array}{c}
y_{1}^{\prime} \\
y_{2}^{\prime}
\end{array}\right]=\left[\begin{array}{c}
y_{1}+y_{2}^{2}+\tilde{x}_{2}^{2} \\
\tilde{x}_{2}+y_{1} \tilde{x}_{2}+\tilde{x}_{1} y_{2}
\end{array}\right], t \in \mathbb{R}
$$

If we choose $V(t, y-\tilde{x})=\|\gamma-\tilde{x}\|^{2}=\langle y-\tilde{x}, y-\tilde{x}\rangle=\left(y_{1}-\tilde{x}_{1}\right)^{2}+\left(y_{2}-\tilde{x}_{2}\right)^{2}$, then $D_{*}^{+} V(t, y-\tilde{x})$ is negative definite as in Example 4.1 we omit the details. Hence, if all the hypotheses of the Theorem 3.2.3 for $q=1$ have been satisfied, then the solution $y$ $\left(t, \tau_{0}, y_{0}\right)$ of (4.4) is initial time difference Lagrange stable with respect to the solution $\tilde{x}\left(t, \tau_{0}, x_{0}\right)$ of (4.3) for $t \geq \tau_{0}, \tau_{0} \in \mathbb{R}$ since we have

$$
D_{*}^{+} V(t, y-\tilde{x}) \leq-2 V(t, y-\tilde{x}) .
$$

We apply Theorem 3.2.3 with the comparison system

$$
u \prime=-2 u, u\left(\tau_{0}\right)=\left\|y_{0}-\tilde{x}_{0}\right\| \geq 0
$$

for $t \geq \tau_{0}$.

Remark 4.3: Boundedness criteria and Lagrange stability of the solution $y\left(t, \tau_{0}, y_{0}\right)$ of the system (2.3) are initial time differences with respect to the solution $x\left(t-\eta, t_{0}, x_{0}\right)$ where $x\left(t, t_{0}, x_{0}\right)$ is the solution of (2.1) for $t \geq \tau_{0} \geq 0, \tau_{0} \in \mathbb{R}$ is inherently dependent on the order to be chosen.

\section{Conclusion}

Lyapunov's second method is a standard technique used in the study of the qualitative behavior of fractional order differential systems with Caputo derivatives along with a comparison results that allows the prediction of behavior of a differential system when the behavior of the null solution of a comparison system is known. The application of Lyapunov's second method in boundedness theory has the advantage of not requiring knowledge of solutions; however, there has been difficulty with this approach when trying to apply it to unperturbed fractional order differential systems and associated perturbed fractional order differential systems with an initial time difference.

The difficulty arises because there is a significant difference between initial time difference boundedness and Lagrange stability and the classical notion of boundedness and Lagrange stability for fractional order differential systems. The classical notions of boundedness and Lagrange stability are with respect to the null solution, but initial time difference boundedness and Lagrange stability are with respect to the unperturbed fractional order differential system where the perturbed fractional order differential system and the unperturbed fractional order differential system differ in initial conditions.

Therefore, in this work, we have dispersed this intricacy and a new comparison result that again gives the null solution a central role in the comparison fractional order differential system. The direct application and generalization of this result in qualitative method have created many paths for continuing research in this direction. 


\section{Author details}

'Department of Mathematics, Gebze Institute of Technology, Gebze-Kocaeli 141-41400, Turkey ${ }^{2}$ Department of Mathematics, Yıldız Technical University, Faculty of Sciences Esenler-Istanbul, 34210, Turkey

\section{Authors' contributions}

The authors have contributed that initial time difference boundedness criteria and Lagrange stability for fractional order differential equation in Caputo's sense are unified with Lyapunov-like functions to establish comparison result. The qualitative behavior of a perturbed fractional order differential equation with Caputo's derivative that differs in initial position and initial time with respect to the unperturbed fractional order differential equation with Caputo's derivative has been investigated. We present a comparison result that again gives the null solution a central role in the comparison fractional order differential equation when establishing initial time difference boundedness criteria and Lagrange stability of the perturbed fractional order differential equation with respect to the unperturbed fractional order differential equation in Caputo's sense.

\section{Competing interests}

The authors declare that they have no competing interests.

Received: 24 December 2010 Accepted: 10 November 2011 Published: 10 November 2011

\section{References}

1. Oldham, KB, Spanier, J: The Fractional Calculus. Academic Press, New York (1974)

2. Samko, S, Kilbas, A, Marichev, O: Fractional Integrals and Derivatives: Theory and Applications. Gordon and Breach 1006 (1993). ISBN 2881248640

3. Baleanu, D, Sadati, SJ, Ghaderi, R., et al: Razumikhin stability theorem for fractional systems with delay. Abstract and Applied Analysis 2010, 1-9 (2010). Article ID 124812

4. Lakshmikantham, V, Leela, S, Vasundhara Devi, J: Theory of Fractional Dynamical Systems. Cambridge Scientific Publishers (2009)

5. Podlubny, I: Fractional Differential Equations. Academic Press, New York (1999)

6. Caputo, M: Linear models of dissipation whose Q is almost independent. II Geophys J R Astronom. 13, 529-539 (1967)

7. Kilbas, AA, Srivastava, HM, Trujillo, JJ: Theory and Applications of Fractional Differential Equations. Elsevier Science BV Amsterdam. (2006)

8. Brauer, F, Nohel, J: The Qualitative Theory of Ordinary Differential Equations. W.A. Benjamin, Inc., New York (1969)

9. Lakshmikantham, V, Leela, S: Differential and Integral Inequalities. Academic Press, New York1 (1969)

10. Lakshmikantham, V, Leela, S, Martynyuk, AA: Stability Analysis of Nonlinear Systems. Marcel Dekker, New York (1989)

11. Li, Y, Chen, YQ, Podlubny, I: Stability of fractional-order nonlinear dynamic systems: Lyapunov direct method and generalized Mittag-Leffler stability. Comput Math Appl. 59(5 SI):1810-1821 (2010)

12. Yakar, C: Boundedness Criteria in Terms of Two Measures with Initial Time Difference. Dynamics of Continuous, Discrete and Impulsive Systems. Series A: Mathematical Analysis. pp. 270-275.Watam Press, Waterloo (2007). DCDIS 14 (S2) 1305

13. Yakar, C, Çiçek, M: Theory, Methods and Applications of Initial Time Difference Bound-edness Criteria and Lagrange Stability in Terms of Two Measures for Nonlinear Systems. Hacettepe Journal of Mathematics and Statistics. 40(2):305-330 (2011)

14. Yakar, C, Shaw, MD: A Comparison Result and Lyapunov Stability Criteria with Initial Time Difference. Dynamics of Continuous, Discrete and Impulsive Systems. Series A: Mathematical Analysis. 12(6):731-741 (2005)

15. Yakar, C, Shaw, MD: Practical stability in terms of two measures with initial time difference. Nonlinear Anal Theory Methods Appl. 71, e781-e785 (2009). doi:10.1016/j.na.2008.11.039

16. Lakshmikantham, V, Vatsala, AS: Differential inequalities with time difference and application. J Inequal Appl. 3, 233-244 (1999)

17. Shaw, MD, Yakar, C: Generalized variation of parameters with initial time difference and a comparison result in term Lyapunov-like functions. Int J Nonlinear Differ Equ Theory Methods Appl. 5, 86-108 (1999)

18. Shaw, MD, Yakar, C: Stability criteria and slowly growing motions with initial time difference. Problems Nonlinear Anal Eng Syst. 1, 50-66 (2000)

19. Yakar, C: Strict Stability Criteria of Perturbed Systems with respect to Unperturbed Systems in term of Initial Time Difference. Proceedings of the Conference on Complex Analysis and Potential Theory. pp. 239-248.World Scientific Publishing (2007)

20. Yakar, C, Shaw, MD: Initial Time Difference Stability in Terms of Two Measures and Variational Comparison Result. Dynamics of Continuous, Discrete and Impulsive Systems. Series A: Mathematical Analysis. 15, 417-425 (2008)

21. Lakshmikanthama, V, Vatsala, AS: Basic theory of fractional differential equations. Nonlinear Anal. 69, 2677-2682 (2008). doi:10.1016/..na.2007.08.042

22. Yakar, C: Fractional differential equations in terms of comparison results and lya-punov stability with initial time difference. Abst Appl Anal 3(762857) (2010). 16

doi:10.1186/1687-1847-2011-54

Cite this article as: Yakar et al:: Boundedness and Lagrange stability of fractional order perturbed system related to unperturbed systems with initial time difference in Caputo's sense. Advances in Difference Equations 2011 2011:54. 